\title{
A New Interpretation of Quantum Mechanics
}

\author{
Shiro Ishikawa \\ Department of Mathematics, Faculty of Science and Technology, Keio University, Hiyoshi, Yokohama, Japan \\ E-mail: ishikawa@math.keio.ac.jp \\ Received July 17, 2011; revised August 1, 2011; accepted August 15, 2011
}

\begin{abstract}
The Copenhagen interpretation is the most authorized interpretation of quantum mechanics, but there are a number of ideas that are associated with the Copenhagen interpretation. It is certain that this fact is not necessarily desirable. Thus, we propose a new interpretation of measurement theory, which is the linguistic aspect (or, the mathematical generalization) of quantum mechanics. Although this interpretation is superficially similar to a part of so-called Copenhagen interpretation, we show that it has a merit to be applicable to both quantum and classical systems. For example, we say that Bell's inequality is broken even in classical systems.
\end{abstract}

Keywords: the Copenhagen Interpretation, Quantum and Classical Measurement Theory, the Law of Large Numbers, Maximum Likelihood Estimation, Kolmogorov Extension Theorem, Wavefunction Collapse, Bell's Inequality

\section{Introduction}

It is well known (cf. [1]) that quantum mechanics is formulated in an operator algebra $B(H)$ (i.e., an operator algebra composed of all bounded linear operators on a Hilbert space $H$ with the norm $\|F\|_{B(H)}=\sup _{\|u\|_{H}=1}$ $\|F u\|_{H}$ ) as follows:

$$
\begin{aligned}
\text { (A) } & \underset{(\text { physics })}{\text { quantum mechanics }} \\
= & \underset{(\text { probabilistic interpretation })}{[\text { quantum measurement }]}[\underset{\text { (kinetic equation) }}{[\text { causality }]}
\end{aligned}
$$

Also, the Copenhagen interpretation due to N. Bohr (et al.) is characterized as the guide to the usage of quantum mechanics $(A)$. Although quantum mechanics $(A)$ with the Copenhagen interpretation is generally accepted as one of the most trustworthy theories in science, it should be noted that there is no definitive statement of the Copenhagen interpretation, that is, there are a number of ideas that are associated with the Copenhagen interpretation. We do not think that this fact is desirable.

Measurement theory (mentioned in Section 2 later or refs. [2-6]) is, by an analogy of the (A), constructed as the mathematical theory formulated in a certain $C^{*}$-algebra $A$ (i.e., a norm closed subalgebra in $B(H), c f$. [7]) as follows:

(B) measurement theory

$$
=\underset{(\text { Rule } 1 \text { in Section } 2)}{[\text { measurement }}]+\underset{(\text { Rule } 2 \text { in Section } 2)}{[\text { causality }]}
$$

Note that this theory $(B)$ is not physics but a kind of language based on the mechanical world view since it is a mathematical generalization of quantum mechanics (A).

When $\mathcal{A}=B_{c}(H)$, the $C^{*}$-algebra composed of all compact operators on a Hilbert space $H$, the $(B)$ is called quantum measurement theory (or, quantum system theory), which can be regarded as the linguistic aspect of quantum mechanics. Also, when $\mathcal{A}$ is commutative (that is, when $\mathcal{A}$ is characterized by $C_{0}(\Omega)$, the $C^{*}$-algebra composed of all continuous complex-valued functions vanishing at infinity on a locally compact Hausdorff space $\Omega$ (cf. [7])), the (B) is called classical measurement theory. Thus, we have the following classification:

(C) measurement theory

$$
=\left\{\begin{array}{c}
\text { quantum measurement theory } \\
\text { (when } \left.\mathcal{A}=B_{C}(H)\right) \\
\text { classical measurement theory } \\
\left(\text { when } \mathcal{A}=C_{0}(\Omega)\right)
\end{array}\right.
$$

That is, this theory covers several conventional system theories (i.e., statistics, dynamical system theory, quantum system theory).

The purpose of this paper is to propose an interpretation of measurement theory $(B)$. Since the $(C)$ says that this interpretation should be common in classical and 
quantum measurement theories, it is also regarded as a new interpretation of quantum mechanics.

\section{Measurement Theory}

Now we shall explain the measurement theory $(B)$. Let $\mathcal{A}(\subseteq B(H))$ be a $C^{*}$-algebra, and let $\mathcal{A}^{*}$ be the dual Banach space of $\mathcal{A}$. That is, $\mathcal{A}^{*}=\{\rho \mid \rho$ is a continuous linear functional on $\mathcal{A}\}$, and the norm $\|\rho\|_{\mathcal{A}^{*}}$ is defined by $\sup \left\{|\rho(F)| \mid F \in \mathcal{A}\right.$ such that $\|F\|_{\mathcal{A}}\left(=\|F\|_{B(H)}\right)$ $\leq 1\}$. Define the mixed state $\rho\left(\in \mathcal{A}^{*}\right)$ such that $\|\rho\|_{\mathcal{A}^{*}}=1$ and $\rho(F) \geq 0$ for all $F \in \mathcal{A}$ such that $F \geq 0$. And define the mixed state space $\mathfrak{S}^{m}\left(\mathcal{A}^{*}\right)$ such that

$$
\mathfrak{S}^{m}\left(\mathcal{A}^{*}\right)=\left\{\rho \in \mathcal{A}^{*} \mid \rho \text { is a mixed state }\right\} \text {. }
$$

A mixed state $\rho\left(\in \mathfrak{S}^{m}\left(\mathcal{A}^{*}\right)\right)$ ) is called a pure state if it satisfies that $\rho=\theta \rho_{1}+(1-\theta) \rho_{2}$ for some $\rho_{1}, \rho_{2}$ $\in \mathfrak{S}^{m}\left(\mathcal{A}^{*}\right)$ and $0<\theta<1$ implies $\rho=\rho_{1}=\rho_{2}$. Put

$$
\mathfrak{S}^{p}\left(\mathcal{A}^{*}\right)=\left\{\rho \in \mathfrak{S}^{m}\left(\mathcal{A}^{*}\right) \mid \rho \text { is a pure state }\right\} .
$$

which is called a state space. It is well known (cf. [7]) that $\mathfrak{S}^{p}\left(B_{c}(H)^{*}\right)=\{|u\rangle\langle u|$ (i.e., the Dirac notation) $\left.\|u\|_{H}=1\right\}$, and $\mathfrak{S}^{p}\left(C_{0}(\Omega)^{*}\right)=\left\{\delta_{\omega_{0}} \mid \delta_{\omega_{0}}\right.$ is a point measure at $\left.\omega_{0} \in \Omega\right\}$, where $\int_{\Omega} f(\omega) \delta_{\omega_{0}}(\mathrm{~d} \omega)=f\left(\omega_{0}\right)$ $\left(\forall f \in C_{0}(\Omega)\right)$. The latter implies that $\mathfrak{S}^{p}\left(C_{0}(\Omega)^{*}\right)$ can be also identified with $\Omega$ such as

$$
\mathfrak{S}^{p}\left(C_{0}(\Omega)^{*}\right) \ni \delta_{\omega_{0}} \leftrightarrow \omega_{0} \in \Omega .
$$

Here, assume that the $C^{*}$-algebra $\mathcal{A}(\subseteq B(H))$ has the identity $I$. This assumption is not unnatural, since, if $I \notin \mathcal{A}$, it suffices to reconstruct the above $\mathcal{A}$ such that it includes $\mathcal{A} \cup\{I\}$. According to the noted idea (cf. [8]) in quantum mechanics, an observable $\mathrm{O}:=(X, \mathcal{F}$, $F)$ in $\mathcal{A}$ is defined as follows:

1) [Field] $X$ is a set, $\mathcal{F}(\subseteq P(X)$, the power set of $X)$ is a field of $X$, that is, " $\Xi_{1}, \Xi_{2} \in \mathcal{F} \Rightarrow \Xi_{1} \cup \Xi_{2} \in \mathcal{F}$ ", " $\Xi \in \mathcal{F} \Rightarrow X \backslash \Xi \in \mathcal{F}$ ".

2) [Finite additivity] $F$ is a mapping from $\mathcal{F}$ to $\mathcal{A}$ satisfying: a): for every $\Xi \in \mathcal{F}, F(\Xi)$ is a nonnegative element in $\mathcal{A}$ such that $0 \leq F(\Xi) \leq I$, b): $F(\varnothing)=0$ and $F(X)=I$, where 0 and $I$ is the 0 -element and the identity in $\mathcal{A}$ respectively. c): for any finite decomposition $\left\{\Xi_{1}, \Xi_{2}, \cdots, \Xi_{n}, \cdots, \Xi_{N}\right\}$ of $\Xi$ (i.e., $\Xi, \Xi_{n} \in F(n=1,2,3, \cdots, N), \cup_{n=1}^{N} \Xi_{n}=\Xi, \Xi_{i} \cap \Xi_{j}$ $=\varnothing(i \neq j))$, it holds that $F(\Xi)=\sum_{n=1}^{N} F\left(\Xi_{n}\right)$.

Remark 1 [Countable additivity] The assumption of the countable additivity (i.e., $F(\Xi)=\lim _{N \rightarrow \infty} \sum_{n=1}^{N} F\left(\Xi_{n}\right)$ in the sense of weak-topology ) may be rather half-fin- ished. If the countable additivity is required, it is, by the reason mentioned in Remark 3 and 6 later, recommended to start from the $W^{*}$-algebra $\overline{\mathcal{A}}$ such that $\mathcal{A} \subseteq \overline{\mathcal{A}} \subseteq$ $B(H)$ as discussed in Appendix later (cf. [5]). However, our interest in this paper is not mathematics but the interpretation of measurement theory. Thus, all arguments will be discussed under the above finite additivity (i.e., 1) and 2)).

With any system $S$, a $C^{*}$-algebra $\mathcal{A}(\subseteq B(H))$ can be associated in which the measurement theory $(B)$ of that system can be formulated. A state of the system $S$ is represented by an element $\rho\left(\in \mathfrak{S}^{p}\left(\mathcal{A}^{*}\right)\right)$ and an $o b$ servable is represented by an observable $\mathrm{O}:=(X, \mathcal{F}, F)$ in $\mathcal{A}$. Also, the measurement of the observable $\mathrm{O}$ for the system $S$ with the state $\rho$ is denoted by $\mathrm{M}_{\mathcal{A}}\left(\mathrm{O}, S_{[\rho]}\right)$ (or more precisely, $\mathrm{M}_{\mathcal{A}}\left(\mathrm{O}:=(X, \mathcal{F}, F), S_{[\rho]}\right)$ ). An observer can obtain a measured value $x(\in X)$ by the measurement $\mathrm{M}_{\mathcal{A}}\left(\mathrm{O}, S_{[\rho]}\right)$.

The Rule 1 presented below is a kind of mathematical generalization of Born's probabilistic interpretation of quantum mechanics $(A)$. And thus, it is a statement without reality.

Rule 1 [Measurement] The probability that a measured value $x(\in X)$ obtained by the measurement $\mathrm{M}_{\mathcal{A}}\left(\mathrm{O}:=(X, \mathcal{F}, F), S_{\left[\rho_{0}\right]}\right)$ belongs to a set $\Xi(\in \mathcal{F})$ is given by $\rho_{0}(F(\Xi))$.

Remark 2 Again note that Rule 1 is a statement without reality (i.e., a kind of incantation or spell). Thus, it is unnecessary (or precisely speaking, impossible) to answer the question: "What is measurement (or, system, state, observable, probability, etc.)?" However, surprisingly, as seen in [2-6] or Section 4 later, man's linguistic competence enables us to use Rule 1. This is essential to our approach to the interpretation of quantum mechanics.

Next, we explain Rule 2 in $(B)$. Let $\mathcal{A}_{1}\left(\subseteq B\left(H_{1}\right)\right)$ and $\mathcal{A}_{2}\left(\subseteq B\left(H_{2}\right)\right)$ be $C^{*}$-algebras. A continuous linear operator $\Phi_{1,2}: \mathcal{A}_{2} \rightarrow \mathcal{A}_{1}$ is called a Markov operator, if it satisfies that 1): $\Phi_{1,2}\left(F_{2}\right) \geq 0$ for any nonnegative element $F_{2}$ in $\mathcal{A}_{2}$, ii): $\Phi_{1,2}\left(I_{2}\right)=I_{1}$, where $I_{k}$ is the identity in $\mathcal{A}_{k},(k=1,2)$. Here note that, for any observable $\mathrm{O}_{2}:=\left(X, \mathcal{F}, F_{2}\right)$ in $\mathcal{A}_{2}$, the $\left(X, \mathcal{F}, \Phi_{1,2} F_{2}\right)$ is an observable in $\mathcal{A}_{1}$, which is denoted by $\Phi_{1,2} \mathrm{O}_{2}$. Also, the dual operator $\Phi_{1,2}^{*}: \mathcal{A}_{1}^{*} \rightarrow \mathcal{A}_{2}^{*}$ clearly satisfies that $\Phi_{1,2}^{*}\left(\mathfrak{S}^{m}\left(\mathcal{A}_{1}^{*}\right)\right) \subseteq \mathfrak{S}^{m}\left(\mathcal{A}_{2}^{*}\right)$.

Let $(T, \leq)$ be a tree, i.e., a partial ordered finite set such that $t_{1} \leq t_{3}$ and $t_{2} \leq t_{3}$ implies $t_{1} \leq t_{2}$ or $t_{2} \leq t_{1}$. Assume that there exists an element $t_{0} \in T$, called the root of $T$, such that $t_{0} \leq t \quad(\forall t \in T)$ holds. Put $T_{\leq}^{2}=\left\{\left(t_{1}, t_{2}\right) \in T^{2} \mid t_{1} \leq t_{2}\right\}$. 
Definition 1 [Markov relation] The family $\left\{\Phi_{t_{1}, t_{2}}\right.$ : $\left.\mathcal{A}_{t_{2}} \rightarrow \mathcal{A}_{t_{1}}\right\}_{\left(t_{1}, t_{2}\right) \in T_{\leq}^{2}}$ is called a Markov relation (due to the Heisenberg picture), if it satisfies the following conditions 1) and 2).

1) With each $t \in T$, a $C^{*}$-algebra $\mathcal{A}_{t}$ is associated.

2) For every $\left(t_{1}, t_{2}\right) \in T_{\leq}^{2}$, a Markov operator $\Phi_{t_{1}, t_{2}}$ : $\mathcal{A}_{t_{2}} \rightarrow \mathcal{A}_{t_{1}}$ is defined. And it satisfies that $\Phi_{t_{1}, t_{2}} \Phi_{t_{2}, t_{3}}$ $=\Phi_{t_{1}, t_{3}}$ holds for any $\left(t_{1}, t_{2}\right),\left(t_{2}, t_{3}\right) \in T_{\leq}^{2}$.

The family of dual operators

$$
\left\{\Phi_{t_{1}, t_{2}}^{*}: \mathfrak{S}^{m}\left(\mathcal{A}_{t_{1}}^{*}\right) \rightarrow \mathfrak{S}^{m}\left(\mathcal{A}_{t_{2}}^{*}\right)\right\}_{\left(t_{1}, t_{2}\right) \in T_{\leq}^{2}}
$$

is called a Markov relation (due to the Schrödinger picture). However, it is not formally used in measurement theory.

Now Rule 2 in the measurement theory $(B)$ is presented as follows:

Rule 2 [Causality] The causality is represented by a Markov relation $\left\{\Phi_{t_{1}, t_{2}}: \mathcal{A}_{t_{2}} \rightarrow \mathcal{A}_{t_{1}}\right\}_{\left(t_{1}, t_{2}\right) \in T_{\leq}^{2}}$.

Remark 3 If an infinite tree $T$ is required, we must start from a $W^{*}$-algebra $\overline{\mathcal{A}}$ ( $c f$. Appendix later). However, in this paper, we, for simplicity, assume the finiteness of $T$. Also, by the same reason mentioned in Remark 2, the question: "What is causality?" is nonsense. What we can do in measurement theory is only to trust in man's linguistic competence.

\section{An Interpretation of Measurement Theory}

The measurement theory $(B)$ asserts "Describe any ordinary phenomenon according to Rules 1 and 2". Still, most readers may be perplexed how to use Rules 1 and 2 since there are various usages. Thus, the following problem is significant.

(D) How should Rules 1 and 2 be used?

Note that reality is not reliable since Rules 1 and 2 are statements without reality. Thus, we want to define the new interpretation such that

(E) the new interpretation is a guide to the most useful (or, powerful, “Occam's razor"-like) usage of Rules 1 and 2.

Now we can present our main assertion in this paper as follows:

$(F)$ The new interpretation defined in the $(E)$ is characterized as the following $\left(F_{1}\right)-\left(F_{3}\right)$.

Here,

$\left(F_{1}\right)$ Consider the dualism composed of "observer" and "system (= measuring object)".

$\left(F_{2}\right)$ Only one measurement is permitted. And thus, the state after a measurement is meaningless since it can not be measured any longer.

$\left(F_{3}\right)$ The causality should be assumed only in the side of system, however, a state never moves. Thus, the Heisenberg picture should be adopted. Also, the observer does not have the space-time. Thus, the question: "When and where is a measured value obtained?" is meaningless,

and so on.

The above may be rather similar to a certain part of so called Copenhagen interpretation. However, note that we do not assume "the state after a measurement (= wavefunction collapse)" and "the Schrödinger picture", which are often investigated in so-called Copenhagen interpretation.

Also, some may consider that the above proposal $(F)$ is too optimistic, since the following question is not yet answered:

(G) Does the most useful usage of Rules 1 and 2 exist? Or, is it determined uniquely?

However, we may be allowed to be optimistic until another most useful usage (or, a powerful rival candidate) will be discovered. In other words, we expect the readers to read the overwhelming predominance of the $(F)$ in the following section or refs. [2-6].

\section{Examples and Remarks}

What we want to assert in this paper is only the $(F)$. Thus, it is desirable that the each one reader verifies the superiority of the $(F)$ in our papers [2-6]. However, in this section we take up some simple examples, which promote the readers' understanding of the $(F)$. Note, for completeness, that all examples are consequences of measurement theory with the interpretation $(F)$.

Example 1 [Parallel measurement, the law of large numbers] For each $k=1,2, \cdots, K$, consider a measurement $\mathrm{M}_{\mathcal{A}_{k}}\left(\mathrm{O}_{k}:=\left(X_{k}, \mathcal{F}_{k}, F_{k}\right), S_{\left[\rho_{k}\right]}\right)$. However, the interpretation $\left(F_{2}\right)$ says that only one measurement is permitted. Thus, we consider the spatial tensor $C^{*}$-algebra $\otimes_{k=1}^{K} \mathcal{A}_{k}\left(\subseteq B\left(\otimes_{k=1}^{K} H_{k}\right)\right)$, and consider the product space $\times_{k=1}^{K} X_{k}$ and the product field $\bigotimes_{k=1}^{K} \mathcal{F}_{k}$, which is defined by the smallest field that contains a family

$$
\left\{x_{k=1}^{K} \Xi_{k} \mid \Xi_{k} \in \mathcal{F}_{k}, k=1,2, \cdots, K\right\} \text {. }
$$

Define the parallel observable

$$
\otimes_{k=1}^{K} \mathrm{O}_{k}=\left(\times_{k=1}^{K} X_{k}, \bigotimes_{k=1}^{K} \mathcal{F}_{k}, \widetilde{F}\right)
$$

in the tensor $C^{*}$-algebra $\otimes_{k=1}^{K} \mathcal{A}_{k}$ such that

$$
\widetilde{F}\left(\times_{k=1}^{K} \Xi_{k}\right)=\otimes_{k=1}^{K} F_{k}\left(\Xi_{k}\right)\left(\forall \Xi_{k} \in \mathcal{F}_{k}, k=1,2, \cdots, K\right) \text {. }
$$

Then, the above $\left\{\mathrm{M}_{\mathcal{A}_{k}}\left(\mathrm{O}_{k}, S_{\left[\rho_{k}\right]}\right)\right\}_{k=1}^{K}$ is represented 
by the parallel measurement $\mathrm{M}_{\otimes_{k=1}^{K} \mathcal{A}_{k}}\left(\otimes_{k=1}^{K} \mathrm{O}_{k} S_{\left[\otimes_{k=1}^{K} \rho_{k}\right]}\right)$, which is also denoted by $\otimes_{k=1}^{K} \mathrm{M}_{\mathcal{A}_{k}}\left(\mathrm{O}_{k}, S_{\left[\rho_{k}\right]}\right)$. Consider a particular case such that, $\mathcal{A}=\mathcal{A}_{k}, \mathrm{O}=(X, \mathcal{F}, F)=$ $\left(X_{k}, \mathcal{F}_{k}, F_{k}\right), \rho=\rho_{k}(\forall k=1,2, \cdots, K)$. Let $\left(x_{1}, x_{2}, \cdots, x_{K}\right)$ $\left(\in X^{K}\right)$ be a measured value by the parallel measurement $\otimes_{k=1}^{K} \mathrm{M}_{\mathcal{A}}\left(\mathrm{O}, S_{[\rho]}\right)$. Then, using Rule 1, we see the law of large numbers, that is, for sufficiently large $K$,

$$
\rho(F(\Xi)) \approx \frac{\#\left\{k=1,2, \cdots, K \mid x_{k} \in \Xi\right\}}{K}(\forall \Xi \in \mathcal{F})
$$

holds (cf. $[2,4])$, where $\#[A]$ is the the number of elements of the set $A$. This is, of course, most fundamental in science. Also, this is the reason that the term "probability" is used in Rule 1 .

Notation $1\left[\mathrm{M}_{\mathcal{A}}\left(\mathrm{O}, S_{[*]}\right)\right]$ It is natural to consider that a measurement $\mathrm{M}_{\mathcal{A}}\left(\mathrm{O}:=(X, \mathcal{F}, F), S_{[\rho]}\right)$ is usually taken in order to know the state $\rho$. Thus, when we want to emphasize that we do not know the state $\rho$, the measurement $\mathrm{M}_{\mathcal{A}}\left(\mathrm{O}, S_{[\rho]}\right)$ is often denoted by $\mathrm{M}_{\mathcal{A}}\left(\mathrm{O}, S_{[*]}\right)$.

Remark 4 [Maximum likelihood estimation in classical measurements] Consider the classical cases in (C). It may be usual to consider that Rule 1 leads the following statement (cf. $[3,4])$, i.e., maximum likelihood estimation in classical measurements:

$(H)$ When we know that a measured value obtained by a measurement $\mathrm{M}_{C_{0}(\Omega)}\left(\mathrm{O}_{1}:=\left(X_{1}, \mathcal{F}_{1}, F_{1}\right), S_{\left[*^{*}\right]}\right)$ belongs to $\Xi_{1}\left(\in \mathcal{F}_{1}\right)$, there is a reason to infer that the unknown state $[*]=\rho_{0}\left(\in \mathfrak{S}^{p}\left(C_{0}(\Omega)^{*}\right)\right)$ where $\rho_{0}\left(\in \mathfrak{S}^{p}\left(C_{0}(\Omega)^{*}\right)\right)$ is defined by $\rho_{0}\left(F_{1}\left(\Xi_{1}\right)\right)=$ $\max _{\rho \in \mathfrak{S}^{p}\left(C_{0}(\Omega)^{*}\right)} \rho\left(F_{1}\left(\Xi_{1}\right)\right)$ if it exists.

Although this $(H)$ is surely handy, note that the $\left(F_{2}\right)$ says that it is illegal to regard the $\rho_{0}$ as the state after the measurement $\mathrm{M}_{C_{0}(\Omega)}\left(\mathrm{O}_{1}, S_{[*]}\right)$. Thus, strictly speaking, the $(H)$ is informal. And thus, it should be reconsidered in Example 2 below.

Example 2 [Maximum likelihood estimation in measurements] By a similar method as the lead of the $(H)$, we can easily see the following statement $(I)$, which should be regarded as the measurement theoretical form of maximum likelihood estimation (cf. Corollary 5.5 in [4]).

(I) When we know that a measured value obtained by a measurement $\mathrm{M}_{\mathcal{A}}\left(\mathrm{O}:=\left(X_{1} \times X_{2}, \mathcal{F}_{1} \otimes \mathcal{F}_{2}, F\right), S_{[*]}\right)$ belongs to $\Xi_{1} \times X_{2}$, there is a reason to infer that the probability that the measured value belongs to $\Xi_{1} \times \Xi_{2}\left(\forall \Xi_{2} \in \mathcal{F}_{2}\right)$ is given by the following conditional probability:

$$
\frac{\rho_{0}\left(F\left(\Xi_{1} \times \Xi_{2}\right)\right)}{\rho_{0}\left(F\left(\Xi_{1} \times X_{2}\right)\right)}
$$

where $\rho_{0}\left(\in \mathfrak{S}^{p}\left(\mathcal{A}^{*}\right)\right)$ is defined by $\rho_{0}\left(F\left(\Xi_{1} \times X_{2}\right)\right)$ $=\max _{\rho \in \mathfrak{S}^{p}\left(\mathcal{A}^{*}\right)} \rho\left(F\left(\Xi_{1} \times X_{2}\right)\right)$ if it exists. Here, note that the $\rho_{0}$ is not the state after the measurement $\mathrm{M}_{\mathcal{A}}\left(\mathrm{O}, S_{[*]}\right)$.

This $(I)$, which also includes quantum cases, is most fundamental in statistics, and thus, we believe (cf. [2-6]) that statistics is one of aspects of measurement theory. For the relation between the informal $(H)$ and the formal (I), see Remark 5 later.

Example 3 [Simultaneous measurement] For each $k=1,2, \cdots, K$, consider a measurement

$$
\mathrm{M}_{\mathcal{A}}\left(\mathrm{O}_{k}:=\left(X_{k}, \mathcal{F}_{k}, F_{k}\right), S_{[\rho]}\right) \text {. }
$$

However, since the $\left(F_{2}\right)$ says that only one measurement is permitted, the $\left\{\mathrm{M}_{\mathcal{A}}\left(\mathrm{O}_{k}, S_{[\rho]}\right)\right\}_{k=1}^{K}$ should be reconsidered in what follows. Under the commutativity condition such that

$$
\begin{aligned}
& F_{i}\left(\Xi_{i}\right) F_{j}\left(\Xi_{j}\right)=F_{j}\left(\Xi_{j}\right) F_{i}\left(\Xi_{i}\right) \\
& \left(\forall \Xi_{i} \in F_{i}, \forall \Xi_{j} \in F_{j}, i \neq j\right),
\end{aligned}
$$

we can define the simultaneous observable $\times_{k=1}^{K} \mathrm{O}_{k}=\left(\times_{k=1}^{K} X_{k}, \bigotimes_{k=1}^{K} \mathcal{F}_{k}, \times_{k=1}^{K} F_{k}\right)$ in $\mathcal{A}$ such that

$$
\begin{aligned}
& \left(\times_{k=1}^{K} F_{k}\right)\left(x_{k=1}^{K} \Xi_{k}\right)=F_{1}\left(\Xi_{1}\right) F_{2}\left(\Xi_{2}\right) \cdots F_{K}\left(\Xi_{K}\right) \\
& \left(\forall \Xi_{k} \in \mathcal{F}_{k}, \forall k=1, \cdots, K\right) .
\end{aligned}
$$

Then, the above $\left\{\mathrm{M}_{\mathcal{A}}\left(\mathrm{O}_{k}, S_{[\rho]}\right)\right\}_{k=1}^{K}$ is, under the commutativity condition (4), represented by the simultaneous measurement $\mathrm{M}_{\mathcal{A}}\left(\times_{k=1}^{K} \mathrm{O}_{k}, S_{[\rho]}\right)$.

Remark 5 [The relation between $(H)$ and $(I)$ ] Consider the $(I)$ in the classical cases, i.e., $\mathcal{A}=C_{0}(\Omega)$. And assume the simultaneous observable $F=F_{1} \times F_{2}$ in (3). Then, putting $\rho_{0}=\delta_{\omega_{0}}$ (i.e., the point measure at $\omega_{0}$ ), we see that

$$
\begin{aligned}
& \text { (3) }=\frac{\left[F_{1}\left(\Xi_{1}\right) \times F_{2}\left(\Xi_{2}\right)\right]\left(\omega_{0}\right)}{\left[F_{1}\left(\Xi_{1}\right) \times F_{2}\left(X_{2}\right)\right]\left(\omega_{0}\right)}=\left[F_{2}\left(\Xi_{2}\right)\right]\left(\omega_{0}\right) \\
& =\rho_{0}\left(F_{2}\left(\Xi_{2}\right)\right)
\end{aligned}
$$

Since this equality holds for any $\mathrm{O}_{2}=\left(X_{2}, \mathcal{F}_{2}, F_{2}\right)$ and any $\Xi_{2} \in \mathcal{F}_{2}$, some may want to regard the $\rho_{0}$ as the state after the measurement $\mathrm{M}_{C_{0}(\Omega)}\left(\mathrm{O}_{1}:=\left(X_{1}, \mathcal{F}_{1}, F_{1}\right), S_{[*]}\right)$ in the $(H)$. Thus, in spite of the $\left(F_{2}\right)$, the $(H)$ may be al- 
lowed in classical cases if the $\rho_{0}$ may be regarded as something represented by the term such as "imaginary state" (cf. [5]). This is the meaning of the informal $(H)$.

Example 4 [How to use Rule 2 (Causality)] Consider a tree $\left(T:=\left\{t_{0}, t_{1}, \cdots, t_{n}\right\}, \leq\right)$ with the root $t_{0}$. This is also characterized by the map $\pi: T /\left\{t_{0}\right\} \rightarrow T$ such that $\pi(t)=\max \{s \in T \mid s<t\}$. Let $\left\{\Phi_{t, t^{\prime}}: \mathcal{A}_{t^{\prime}} \rightarrow \mathcal{A}_{t}\right\}_{\left(t, t^{\prime}\right) \in T_{s}^{2}}$ be a Markov relation, which is also represented by $\left\{\Phi_{\pi(t), t}: \mathcal{A}_{t} \rightarrow \mathcal{A}_{\pi(t)}\right\}_{t \in T /\left\{t_{0}\right\}}$. Let an observable $\mathrm{O}_{t}:=$ $\left(X_{t}, \mathcal{F}_{t}, F_{t}\right)$ in the $\mathcal{A}_{t}$ be given for each $t \in T$. And let $\rho_{0} \in \mathfrak{S}^{p}\left(\mathcal{A}_{t_{0}}^{*}\right)$. Consider "measurements" such as

$(J)$ for each $t \in T$, take a measurement of an observable $\mathrm{O}_{t}$ for the system with a "moving state"

$$
\left[\rho_{0} ;\left\{\Phi_{\pi(t), t}^{*}: \mathbb{S}^{m}\left(\mathcal{A}_{\pi(t)}^{*}\right) \rightarrow \mathbb{S}^{m}\left(\mathcal{A}_{t}^{*}\right)\right\}_{t \in T /\left\{t_{0}\right\}}\right],
$$

where the meaning of "moving state" is not clear yet. Recalling that the $\left(F_{3}\right)$ says that a state never moves, we consider the meaning of the $(\mathrm{J})$ as follows: For each $s \in T$, put $T_{s}=\{t \in T \mid t \geq s\}$. And define the observable $\widehat{\mathrm{O}}_{s}=\left(\times_{t \in T_{s}} X_{t}, \bigotimes_{\in T_{s}} \mathcal{F}_{t}, \widehat{F}_{s}\right)$ in $\mathcal{A}_{s}$ (due to Heisenberg picture) as follows:

$$
\widehat{\mathrm{O}}_{s}= \begin{cases}\mathrm{O}_{s} & (\text { if } \mathrm{s} \in T / \pi(T)) \\ \mathrm{O}_{s} \times\left(\times_{t \in \pi^{-1}(\{s\})} \Phi_{\pi(t), t} \widehat{\mathrm{O}}_{t}\right) & (\text { if } \mathrm{s} \in \pi(T))\end{cases}
$$

if the commutativity condition holds (i.e., if the simultaneous observable $\mathrm{O}_{s} \times\left(\times_{t \in \pi^{-1}(\{s\})} \Phi_{\pi(t), t} \widehat{\mathrm{O}}_{t}\right)$ exists) for each $s \in \pi(T)$. Using (6) iteratively, we can finally obtain the observable $\widehat{\mathrm{O}}_{t_{0}}$ in $\mathcal{A}_{t_{0}}$ Thus the above $(J)$ is represented by the measurement $\mathrm{M}_{\mathcal{A}_{t_{0}}}\left(\widehat{\mathrm{O}}_{t_{0}}, S_{\left[\rho_{0}\right]}\right)$. This also satisfies the $\left(F_{2}\right)$.

Remark 6 [Kolmogorov extension theorem] In the general cases such that countable additivity and infinite $T$ (in Remarks 1 and 3) are required, the existence of the above $\widehat{\mathrm{O}}_{t_{0}}$ is, by using the Kolmogorov extension theorem in probability theory [9], proved in the $W^{*}$ algebraic formulation (cf. $[4,5]$ and Appendix later). We think that this fact is evidence that the interpretation $\left(F_{2}\right)$ is hidden behind the utility of the Kolmogorov extension theorem. Recall the following well-known statement that always appears in the beginning of probability theory:

(K) Let $(X, \mathcal{F}, P)$ be a probability space. Then, the probability that an event $\Xi(\in \mathcal{F})$ occurs is given by $P(\Xi)$,

which, as well as Rule 1, is a statement without reality. We consider that the Kolmogorov extension theorem is regarded as one of the finest answers to the problem: How should the statement $(K)$ be used? That is, in mathematical probability theory, the answer is presented as the form of a basic theorem (i.e., the Kolmogorov extension theorem). On the other hand, in measurement theory, the problem $(D)$ is answered by the interpretation (F).

Remark 7 [Wavefunction collapse] Again reconsider the (J) in the simplest case that $T=\left\{t_{0}, t_{1}\right\}, \pi\left(t_{1}\right)=t_{0}$. Taking a measurement $\mathrm{M}_{\mathcal{A}_{t_{0}}}\left(\mathrm{O}_{t_{0}}, S_{\left[\rho_{0}\right]}\right)$, we know that the measured value belongs to $\Xi_{0}\left(\in \mathcal{F}_{t_{0}}\right)$. Then, it may be usual to consider that a certain wavefunction collapse happens by the measurement, that is, $\mathfrak{S}^{p}\left(\mathcal{A}_{t_{0}}^{*}\right) \ni$ $\rho_{0} \mapsto \rho_{0}^{\Xi_{0}} \in \mathfrak{S}^{p}\left(\mathcal{A}_{t_{0}}^{*}\right)$. And continuously, we take a measurement $\left.\mathrm{M}_{\mathcal{A}_{t_{0}}}\left(\Phi_{t_{0}, t_{1}} \mathrm{O}_{t_{1}}, S_{\left[\rho_{0}^{\Xi_{0}}\right.}\right]\right)$. Here, the probability that a measured value belongs to $\Xi_{1}\left(\in \mathcal{F}_{t_{1}}\right)$ is, by Rule 1, given by $\rho_{0}^{\Xi_{0}}\left(\Phi_{t_{0}, t_{1}} F_{1}\left(\Xi_{1}\right)\right)$. However, this $\rho_{0}^{\Xi_{0}}\left(\Phi_{t_{0}, t_{1}} F_{1}\left(\Xi_{1}\right)\right)$ must be equal to the conditional

$$
\text { probability } \frac{\rho_{0}\left(\widehat{F}\left(\Xi_{0} \times \Xi_{1}\right)\right)}{\rho_{0}\left(\widehat{F}\left(\Xi_{0} \times X_{1}\right)\right)}
$$

if the commutativity condition holds (i.e., the simultaneous observable

$$
\begin{aligned}
\widehat{\mathrm{O}}_{t_{0}} & =\mathrm{O}_{t_{0}} \times \Phi_{t_{0}, t_{1}} \mathrm{O}_{t_{1}} \\
& =\left(X_{t_{0}} \times X_{t_{1}}, \mathcal{F}_{t_{0}} \otimes \mathcal{F}_{t_{1}}, \widehat{F}:=F_{t_{0}} \times \Phi_{t_{0}, t_{1}} F_{t_{1}}\right)
\end{aligned}
$$

exists). This implies that it suffices to consider only the measurement $\mathrm{M}_{A_{t_{0}}}\left(\mathrm{O}_{t_{0}} \times \Phi_{t_{0}, t_{1}} \mathrm{O}_{t_{1}}, S_{\left[\rho_{0}\right]}\right)$. That is, two measurements $\mathrm{M}_{\mathcal{A}_{t_{0}}}\left(\mathrm{O}_{t_{0}}, S_{\left[\rho_{0}\right]}\right)$ and

$$
\mathrm{M}_{\mathcal{A}_{t_{0}}}\left(\Phi_{t_{0}, t_{1}} \mathrm{O}_{t_{1}}, S_{\left[\rho_{0}^{\Xi_{0}}\right]}\right)
$$

are not needed. Also, if the commutativity condition is ignored in the argument of the wavefunction collapse, it is doubtful.

Example 5 [Bell's inequality] According to [11], we shall study the following steps [1-3] in measurement theory.

[Step 1]: Put $X=\{-1,1\}$. Let $a=\alpha_{1}+\alpha_{2} \sqrt{-1}$ and $b=\beta_{1}+\beta_{2} \sqrt{-1}$ be complex numbers such that $|a| \equiv$ $\sqrt{\left|\alpha_{1}\right|^{2}+\left|\alpha_{2}\right|^{2}}=1$ and $|b| \equiv \sqrt{\left|\beta_{1}\right|^{2}+\left|\beta_{2}\right|^{2}}=1$. Consider a probability space $\left(X^{2}, \mathcal{P}\left(X^{2}\right), v_{a b}\right)$ such that

$$
\begin{aligned}
& v_{a b}(\{(1,1)\})=v_{a b}(\{(-1,-1)\})=\left(1-\alpha_{1} \beta_{1}-\alpha_{2} \beta_{2}\right) / 4 \\
& v_{a b}(\{(1,-1)\})=v_{a b}(\{(-1,1)\})=\left(1+\alpha_{1} \beta_{1}+\alpha_{2} \beta_{2}\right) / 4 .
\end{aligned}
$$

Define and calculate the correlation function $P(a, b)$ such that 


$$
P(a, b) \equiv \sum_{\left(x_{1}, x_{2}\right) \in X \times X} x_{1} \cdot x_{2} v_{a b}\left(\left\{\left(x_{1}, x_{2}\right)\right\}\right)=-\alpha_{1} \beta_{1}-\alpha_{2} \beta_{2}
$$

Our problem is as follows.

(L) Find a measurement

$\mathrm{M}_{\mathcal{A}}\left(\mathrm{O}_{a b}:=\left(X^{2}, \mathcal{P}\left(X^{2}\right), F_{a b}\right), S_{\left[\rho_{0}\right]}\right)$ in a $C^{*}$-algebra

$\mathcal{A}$ such that $v_{a b}(\Xi)=\rho_{0}\left(F_{a b}(\Xi)\right)$

$$
\left(\forall \Xi \in P\left(X^{2}\right)\right) \text {. }
$$

This will be answered in the following step [2].

[Step: 2]. In what follows, we shall investigate the $(L)$ in two cases (i.e., quantum case $\left[\mathcal{A}=B\left(\mathbb{C}^{2} \otimes \mathbb{C}^{2}\right)\right]$ and classical case $\left.\left[\mathcal{A}=C_{0}(\Omega \times \Omega)\right]\right)$.

1): Quantum case

$$
\begin{gathered}
{\left[\mathcal{A}=B\left(\mathbb{C}^{2}\right) \otimes B\left(\mathbb{C}^{2}\right)=B\left(\mathbb{C}^{2} \otimes \mathbb{C}^{2}\right) \quad(c f .[10,11])\right]} \\
\text { Put } \quad e_{1}=\left[\begin{array}{l}
1 \\
0
\end{array}\right], \quad e_{2}=\left[\begin{array}{l}
0 \\
1
\end{array}\right] \quad\left(\in \mathbb{C}^{2}\right) .
\end{gathered}
$$

For each $c \in\{a, b\}$, define the observables

$$
\mathrm{O}_{c} \equiv\left(X, \mathcal{P}(X), G_{c}\right)
$$

in $B\left(\mathbb{C}^{2}\right)$ such that

$$
G_{c}(\{1\})=\frac{1}{2}\left[\begin{array}{ll}
1 & \bar{c} \\
c & 1
\end{array}\right], \quad G_{c}(\{-1\})=\frac{1}{2}\left[\begin{array}{cc}
1 & -\bar{c} \\
-c & 1
\end{array}\right] .
$$

Further consider the quantum system composed of two particles formulated in $B\left(\mathbb{C}^{2} \otimes \mathbb{C}^{2}\right)$. Put $\rho_{s}=\left|\psi_{s}\right\rangle\left\langle\psi_{s}\right|$ and $\rho_{0}=\left|\psi_{0}\right\rangle\left\langle\psi_{0}\right|\left(\in \mathfrak{S}^{p}\left(B\left(\mathbb{C}^{2} \otimes \mathbb{C}^{2}\right)^{*}\right)\right)$, where $\psi_{s}=$ $\left(e_{1} \otimes e_{2}-e_{2} \otimes e_{1}\right) / \sqrt{2}$ and $\psi_{0}=e_{1} \otimes e_{1}$. Consider the unitary operator $U\left(\in B\left(\mathbb{C}^{2} \otimes \mathbb{C}^{2}\right)\right.$ such that $U \psi_{0}=\psi_{s}$.

Define the observable

$$
\mathrm{O}_{a b}=\left(X^{2}, \mathcal{P}\left(X^{2}\right), F_{a b}:=U^{*}\left(G_{a} \otimes G_{b}\right) U\right)
$$

in $B\left(\mathbb{C}^{2} \otimes \mathbb{C}^{2}\right)$. Thus we get a measurement

$$
\mathrm{M}_{B\left(\mathbb{C}^{2} \otimes \mathbb{C}^{2}\right)}\left(\mathrm{O}_{a b}, S_{\left[\rho_{0}\right]}\right) \text { in } B\left(\mathbb{C}^{2} \otimes \mathbb{C}^{2}\right) \text {. }
$$

This clearly satisfies the $(L)$ since we easily calculate that, for each $\left(x_{1}, x_{2}\right) \in X^{2}$,

$$
\begin{aligned}
& \rho_{0}\left(F_{a b}\left(\left\{\left(x_{1}, x_{2}\right)\right\}\right)\right) \\
& =\left\langle\psi_{0}, F_{a b}\left(\left\{\left(x_{1}, x_{2}\right)\right\}\right) \psi_{0}\right\rangle \\
& =\left\langle\psi_{s},\left(G_{a}\left(\left\{x_{1}\right\}\right) \otimes G_{b}\left(\left\{x_{2}\right\}\right)\right) \psi_{s}\right\rangle \\
& =v_{a b}\left(\left\{\left(x_{1}, x_{2}\right)\right\}\right) .
\end{aligned}
$$

2): Classical case $\left[A=C_{0}(\Omega) \otimes C_{0}(\Omega)=C_{0}(\Omega \times \Omega)\right]$ Let $\omega_{0}\left(=\left(\omega_{0}^{\prime}, \omega_{0}^{\prime \prime}\right)\right) \in \Omega \times \Omega$, and put $\rho_{0}=\delta_{\omega_{0}}$

$$
\left(\in \mathfrak{S}^{p}\left(C_{0}(\Omega \times \Omega)^{*}\right),\right.
$$

And define the observable $\mathrm{O}_{a b}:=\left(X^{2}, \mathcal{P}\left(X^{2}\right), F_{a b}\right)$ in $C_{0}(\Omega \times \Omega)$ such that

$$
\left[F_{a b}\left(\left\{\left(x_{1}, x_{2}\right)\right\}\right)\right]\left(\omega_{0}\right)=v_{a b}\left(\left\{\left(x_{1}, x_{2}\right)\right\}\right) .
$$

Thus we get a measurement $\mathrm{M}_{C_{0}(\Omega \times \Omega)}\left(\mathrm{O}_{a b}, S_{\left[\delta_{\omega_{0}}\right]}\right)$, which clearly satisfies the $(L)$.

[Step: 3]. For each $k=1,2$, let $a^{k}\left(=\alpha_{1}^{k}+\alpha_{2}^{k} \sqrt{-1}\right)$ and $b^{k}\left(=\beta_{1}^{k}+\beta_{2}^{k} \sqrt{-1}\right)$ be complex numbers such that $\left|a^{k}\right|=\left|b^{k}\right|=1$. Further, consider the parallel measurement $\otimes_{i, j=1,2} \mathrm{M}_{\mathcal{A}}\left(\mathrm{O}_{a^{i} b^{j}}:=\left(X^{2}, \mathcal{P}\left(X^{2}\right), F_{a^{i} b^{j}}\right), S_{\left[\rho_{0}\right]}\right)$ in the tensor $C^{*}$-algebra $\otimes_{i, j=1,2} \mathcal{A}$, by which the measured value $x\left(\in X^{8}\right)$ is obtained as follows:

$$
\begin{aligned}
& x=\left(\left(x_{1}^{11}, x_{2}^{11}\right),\left(x_{1}^{12}, x_{2}^{12}\right),\left(x_{1}^{21}, x_{2}^{21}\right),\left(x_{1}^{22}, x_{2}^{22}\right)\right) \\
& \in \underset{i, j=1,2}{\times} X^{2}
\end{aligned}
$$

Here, the (7) says that, for each $i, j=1,2$,

$$
\begin{aligned}
P\left(a^{i}, b^{j}\right) & =\sum_{\left(x_{1}^{i j}, x_{2}^{i j}\right) \in X \times X} x_{1}^{i j} \cdot x_{2}^{i j} \rho_{0}\left(F_{a^{i} b^{j}}\left(\left\{\left(x_{1}^{i j}, x_{2}^{i j}\right)\right\}\right)\right) \\
& =-\alpha_{1}^{i} \beta_{1}^{j}-\alpha_{2}^{i} \beta_{2}^{j}
\end{aligned}
$$

Thus, putting

$$
a^{1}=\sqrt{-1}, b^{1}=\frac{1+\sqrt{-1}}{\sqrt{2}}, a^{2}=1, b^{2}=\frac{1-\sqrt{-1}}{\sqrt{2}},
$$

we calculate that

$$
\left|P\left(a^{1}, b^{1}\right)-P\left(a^{1}, b^{2}\right)\right|+\left|P\left(a^{2}, b^{1}\right)+P\left(a^{2}, b^{2}\right)\right|=2 \sqrt{2}
$$

Therefore, we can conclude that

$(M)$ Bell's inequality (i.e., a certain inequality such as "the left-hand side of $(8) " \leq 2,(c f .[10,11]))$ is broken in classical systems as well as in quantum systems.

This may be somewhat significant since it says that the non-commutativity of $B\left(\mathbb{C}^{2}\right)$ is not necessarily indispensable for the (8). Although the above discussion in the steps [1-3] is easy and similar to that of [11], it should be noted that we can not assert the $(M)$ if we do not have measurement theory (particularly, classical measurement theory) with the interpretation $(F)$. 


\section{Conclusions}

Since we advanced from quantum mechanics (i.e., the mathematical formulation of Heisenberg's uncertainty principle; [12]) to classical measurement theory, at first we had no way except relying on so-called Copenhagen interpretation in our investigations. That is, we investigated classical measurement theory [2-6] without the clear answer to the problem:

$(N)$ What is the Copenhagen interpretation? What is "interpretation"? Or, how should Rules 1 and 2 be used?

However, in this paper we assert that we can master measurement theory thanks to man's linguistic competence if we know the interpretation $(F)$, which may be characterized as the essence extracted from various ideas in so-called Copenhagen interpretation.

Although N. Bohr said, in the Bohr-Einstein debates $[13,14]$, that the interpretation of a physical theory has to rely on an experimental practice, we consider that the reality should be abandoned if we hope that measurement theory includes classical measurements. In this sense, we agree with A. Einstein, who never accepted the Copenhagen interpretation as physics. That is, in spite of Bohr's realistic view, we propose the following linguistic view:

$(O)$ In the beginning was the language called measurement theory (with the interpretation $(F)$ ). And, for example, quantum mechanics can be fortunately described in this language. And moreover, almost all scientists have already mastered this language partially and informally since statistics (at least, its basic part) is characterized as one of aspects of measurement theory (cf. [2-6]).

If it is so, measurement theory may be a miraculous language, which is common in almost all fields of science. We want to consider that this miracle was originally discovered by J. von Neumann in his famous book [1]. That is because we think that measurement theory (with the interpretation $(F)$ ) is the necessary consequence of his Hilbert space formulation.

Although we believe that the interpretation $(F)$ is the unique answer to the problem $(D)$, this should be of course examined seriously. Thus, we hope that our proposal $(F)$, which is the common interpretation of classical and quantum systems, will be discussed from various viewpoints.

\section{Acknowledgements}

The author wishes to acknowledge Prof. S. Koizumi in
Keio University for valuable suggestions.

\section{References}

[1] J. von Neumann, "Mathematical Foundations of Quantum Mechanics," Springer Verlag, Berlin, 1932.

[2] S. Ishikawa, "A Quantum Mechanical Approach to Fuzzy Theory," Fuzzy Sets and Systems, Vol. 90, No. 3, 1997, pp. 277-306. doi:10.1016/S0165-0114(96)00114-5

[3] S. Ishikawa, "Statistics in Measurements," Fuzzy Sets and Systems, Vol. 116, No. 2, 2000, pp. 141-154. doi:10.1016/S0165-0114(98)00280-2

[4] S. Ishikawa, "Mathematical Foundations of Measurement Theory," Keio University Press Inc., 2006, 335 Pages. http://www.keio-up.co.jp/kup/mfomt/).

[5] S. Ishikawa, "A New Formulation of Measurement Theory," Far East Journal of Dynamical Systems, Vol. 10, No. 1, 2008, pp. 107-117.

[6] K. Kikuchi, S. Ishikawa, "Psychological tests in measurement theory," Far East Journal of Theoretical Statistics, Vol. 32, No. 1, 2010, pp. 81-99.

[7] S. Sakai, "C*-Algebras and $\mathrm{W}^{*}$-Algebras," Ergebnisse der Mathematik und ihrer Grenzgebiete (Band 60), SpringerVerlag, Berlin, 1971.

[8] E. B. Davies, "Quantum Theory of Open Systems," Academic Press, Cambridge, 1976.

[9] A. Kolmogorov, "Foundations of the Theory of Probability (Translation)," Chelsea Publishing Co., New York, 1950.

[10] J. S. Bell, "On the Einstein-Podolosky-Rosen Paradox," Physics, Vol. 1, 1966, pp. 195-200.

[11] F. Selleri, "Die Debatte um die Quantentheorie," Friedr. Vieweg \& Sohn Verlagsgesellscvhaft MBH, Braunschweig, 1983.

[12] S. Ishikawa, "Uncertainty Relation in Simultaneous Measurements for Arbitrary Observables," Reports on Mathematical Physics, Vol. 9, 1991, pp. 257-273. doi:10.1016/0034-4877(91)90046-P

[13] A. Einstein, B. Podolosky and N. Rosen, "Can Quantum-Mechanical Description of Physical Reality Be Considered Complete?" Physical Review, Vol. 47, No. 10, 1935, pp. 777-780. doi:10.1103/PhysRev.47.777

[14] N. Bohr, "Can Quantum-Mechanical Description of Physical Reality Be Considered Complete?" Physical Review, Vol. 48, 1935, pp. 696-702. doi:10.1103/PhysRev.48.696 


\section{Appendix: $W^{*}$-algebraic formulation}

The $C^{*}$-algebraic formulation (mentioned in this paper) is fundamental and essential in measurement theory. However, as mentioned in Remarks 1 and 3 , the $W^{*}$-algebraic formulation ( $c f$. [5]) is, from the mathematical point of view, more handy than the $C^{*}$-algebraic formulation (just like the Lebesgue integral is more handy than the Riemann integral). Thus we think that each of two formulations has its merits and demerits. In what follows, according to [5], in which there is a part that should be corrected, we shall add the $W^{*}$-algebraic formulation.

Consider the pair $[\mathcal{A}, \overline{\mathcal{A}}]_{B(H)}$, called a basic structure. Here, $\mathcal{A}(\subseteq B(H))$ is a $C^{B}$-algebra, and $\overline{\mathcal{A}}(\mathcal{A} \subseteq \overline{\mathcal{A}} \subseteq$ $B(H))$ is a particular $C^{*}$-algebra (called a $W^{*}$-algebra) such that $\overline{\mathcal{A}}$ is the weak closure of $\mathcal{A}$ in $B(H)$. Let $\overline{\mathcal{A}}_{*}$ be the pre-dual Banach space, whose existence is assured (cf. [7]).

For example, we see (cf. [7]) that, when $\mathcal{A}=B_{c}(H)$,

1) $\mathcal{A}^{*}=$ "trace class", $\overline{\mathcal{A}}=B(H), \overline{\mathcal{A}}_{*}=$ "trace class".

Also, when $\mathcal{A}=C_{0}(\Omega)$,

2) $\mathcal{A}^{*}=$ "the space of all signed measures on $\Omega$ ", $\overline{\mathcal{A}}=L^{\infty}(\Omega, v)\left(\subseteq B\left(L^{2}(\Omega, v)\right)\right), \quad \overline{\mathcal{A}}_{*}=L^{1}(\Omega, v)$, where $v$ is some measure on $\Omega$ (cf. [7]).

For instance, in the above 2) we must clarify the meaning of the "value" of $F\left(\omega_{0}\right)$ for $F \in L^{\infty}(\Omega, v)$ and $\omega_{0} \in \Omega$. This is easily done as follows. Let $\mathfrak{S}^{p}\left(\mathcal{A}^{*}\right)$ be as in (2). An element $F(\in \overline{\mathcal{A}})$ is said to be essentially continuous at $\rho_{0}\left(\in \mathfrak{S}^{p}\left(\mathcal{A}^{*}\right)\right)$, if there uniquely exists a complex number $\alpha$ such that

(*) $^{*}$ if $\rho\left(\in \overline{\mathcal{A}}_{*},\|\rho\|_{\overline{\mathcal{A}}_{*}}=1\right)$ converges to $\rho_{0}\left(\in \mathfrak{S}^{p}\left(\mathcal{A}^{*}\right)\right)$ in the sense of weak topology of $\mathcal{A}^{*}$ (i.e., $\left.\rho(G) \rightarrow \rho_{0}(G) \quad(\forall G \in \mathcal{A}(\subseteq \overline{\mathcal{A}}))\right)$, then $\rho(F)$ converges to $\alpha$.

And the value of $\rho_{0}(F)$ is defined by the $\alpha$.

An observable $\mathrm{O}:=(X, \mathcal{F}, F)$ in $\overline{\mathcal{A}}$ is defined as follows:

1) $[\sigma$-field $] X$ is a set, $\mathcal{F}(\subseteq \mathcal{P}(X)$, the power set of $X$ ) is a $\sigma$-field of $X$, that is, " $\Xi_{1}, \Xi_{2}, \cdots \in F \Rightarrow$ $\cup_{n=1}^{\infty} \Xi_{n} \in F ", " \Xi \in F \Rightarrow X / \Xi \in F$.

2) [Countable additivity] $F$ is a mapping from $\mathcal{F}$ to $\overline{\mathcal{A}}$ satisfying: a): for every $\Xi \in \mathcal{F}, F(\Xi)$ is a non-negative element in $\overline{\mathcal{A}}$ such that $0 \leq F(\Xi) \leq I, \mathrm{~b})$ : $F(\varnothing)=0$ and $F(X)=I$, where 0 and $I$ is the 0 -element and the identity in $\overline{\mathcal{A}}$ respectively. c): for any countable decomposition $\left\{\Xi_{1}, \Xi_{2}, \cdots, \Xi_{n}, \cdots\right\}$ of $\Xi$ (i.e., $\Xi, \Xi_{n} \in \mathcal{F}(n=1,2,3, \cdots), \quad \cup_{n=1}^{\infty} \Xi_{n}=\Xi, \quad \Xi_{i} \cap \Xi_{j}=\varnothing$ $(i \neq j))$, it holds that $F(\Xi)=\sum_{n=1}^{\infty} F\left(\Xi_{n}\right)$ in the sense of weak ${ }^{*}$ topology in $\overline{\mathcal{A}}$.

Now we can present Rule 1 in the $W^{*}$-algebraic formulation as follows.

Rule 1' [Measurement] The probability that a measured value $x(\in X)$ obtained by the measurement $\mathrm{M}_{\overline{\mathcal{A}}}\left(\mathrm{O}:=(X, \mathcal{F}, F), S_{\left[\rho_{0}\right]}\right)$ belongs to a set $\Xi(\in \mathcal{F})$ is given by $\rho_{0}(F(\Xi))$ if $F(\Xi)$ is essentially continuous at $\rho_{0}\left(\in \mathfrak{S}^{p}\left(\mathcal{A}^{*}\right)\right)$.

Next, we explain Rule 2. Let $\left[\mathcal{A}_{1}, \overline{\mathcal{A}}_{1}\right]_{B\left(H_{1}\right)}$ and $\left[\mathcal{A}_{2}, \overline{\mathcal{A}}_{2}\right]_{B\left(\mathrm{H}_{2}\right)}$ be basic structures. A continuous linear operator $\Phi_{1,2}: \overline{\mathcal{A}}_{2}$ (with weak ${ }^{*}$ topology) $\rightarrow \overline{\mathcal{A}}_{1}$ (with weak $^{*}$ topology) is called a Markov operator, if it satisfies that 1): $\Phi_{1,2}\left(F_{2}\right) \geq 0$ for any non-negative element $F_{2}$ in $\left.\overline{\mathcal{A}}_{2}, 2\right): \Phi_{1,2}\left(I_{2}\right)=I_{1}$, where $I_{k}$ is the identity in $\overline{\mathcal{A}}_{k},(k=1,2)$. Here note that, for any observable $\mathrm{O}_{2}:=\left(X, \mathcal{F}, F_{2}\right)$ in $\overline{\mathcal{A}}_{2}$, the $\left(X, \mathcal{F}, \Phi_{1,2} F_{2}\right)$ is an observable in $\mathcal{A}_{1}$.

Remark 8 In addition to the above 1) and 2), it may be natural to assume that $\Phi_{1,2}\left(\mathcal{A}_{2}\right) \subseteq \mathcal{A}_{1}$ and

$$
\sup \left\{\left\|\Phi_{1,2}\left(F_{2}\right)\right\|_{\mathcal{A}_{1}} \mid F_{2} \in \mathcal{A}_{2} \text { such that }\left\|F_{2}\right\|_{\mathcal{A}_{2}} \leq 1\right\}=1 \text {. }
$$

However, from the mathematical point of view, this assumption is not necessarily needed.

Let $(T, \leq)$ be a tree, i.e., a partial ordered set such that " $t_{1} \leq t_{3}$ and $t_{2} \leq t_{3}$ " implies " $t_{1} \leq t_{2}$ or $t_{2} \leq t_{1}$ ". Here, note that $T$ is not necessarily finite.

Assume the completeness of the ordered set $T$. That is, for any subset $T^{\prime}(\subseteq T)$ bounded from below (i.e., there exists $t^{\prime}(\in T)$ such that $\left.t^{\prime} \leq t \quad\left(\forall t \in T^{\prime}\right)\right)$, there uniquely exists an element $\inf \left(T^{\prime}\right) \in T$ satisfying the following conditions, 1): $\left.\inf \left(T^{\prime}\right) \leqq t\left(\forall t \in T^{\prime}\right), 2\right)$ : if $s \leqq t\left(\forall t \in T^{\prime}\right)$, then $s \leqq \inf \left(T^{\prime}\right)$.

Definition 1' [Markov relation] The family $\left\{\Phi_{t_{1}, t_{2}}: \overline{\mathcal{A}}_{t_{2}} \rightarrow \overline{\mathcal{A}}_{t_{1}}\right\}_{\left(t_{1}, t_{2}\right) \in T_{\leq}^{2}}$ is called a Markov relation (due to the Heisenberg picture), if it satisfies the following conditions 1) and 2).

1) With each $t \in T$, a basic structure $\left[\mathcal{A}_{t}, \overline{\mathcal{A}}_{t}\right]_{B\left(H_{t}\right)}$ is associated.

2) For every $\left(t_{1}, t_{2}\right) \in T_{\leq}^{2}$, a Markov operator $\Phi_{t_{1}, t_{2}}$ : $\overline{\mathcal{A}}_{t_{2}} \rightarrow \overline{\mathcal{A}}_{t_{1}}$ is defined. And it satisfies that $\Phi_{t_{1}, t_{2}} \Phi_{t_{2}, t_{3}}$ $=\Phi_{t_{1}, t_{3}}$ holds for any $\left(t_{1}, t_{2}\right),\left(t_{2}, t_{3}\right) \in T_{\leq}^{2}$.

Now Rule 2 is presented as follows:

Rule 2' [Causality] The causality is represented by a Markov relation $\left\{\Phi_{t_{1}, t_{2}}: \overline{\mathcal{A}}_{t_{2}} \rightarrow \overline{\mathcal{A}}_{t_{1}}\right\}_{\left(t_{1}, t_{2}\right) \in T_{\leq}^{2}}$. 